\title{
Reducing of Frequency Reuse Distance using Sectoring in Mobile Communication
}

\author{
Debabrata Sarddar ${ }^{1},{ }^{*}$ Priyajit $\mathrm{Sen}^{2}$, Rajat Pandit ${ }^{3}$ and Sougata Chakraborty ${ }^{4}$ \\ ${ }^{1}$ Assistant professor, Department of Computer Science and Engineering, \\ University of Kalyani, Kalyani \\ ${ }^{*}$ Student of Master of Technology, Department of Computer Science and \\ Engineering, University of Kalyani, Kalyani \\ ${ }^{3}$ Assistant professor, Department of Computer Science, West Bengal State \\ University, West Bengal \\ ${ }^{4}$ Senior System Engineer, IBM India Private Limited, Kolkata, West Bengal \\ dsarddar1@gmail.com,priyajit91@gmail.com,rajatpandit123@gmail.com, \\ me.sougata.chakraborty@gmail.com
}

\begin{abstract}
Radio covered area of a base station is called cell. These are served by at least one fixed-location transceiver, known as a cell site or base station. In a radio network, different set of frequencies are used by each cell from their neighboring cells to avoid clash and serve guaranteed bandwidth within each cell. Interference can be minimized by maintaining a certain distance between cells that uses the same frequencies. Hence, this distance can be shortened without disturbing the cell reuse pattern. As the sizes of the cells are reduced, the identical frequencies can be used in various cells, which in turn mean more subscribers can be accommodated on the system. In our proposed work we use the concept of sectoring. Using sectoring a cells area is decreased depending on the type of sectoring used. As area of the cell gets reduced the power needed to cover the area also gets reduced and with transmitter antenna's power gets reduces so as the reuse distance of cell. So reuse distance is proportional to transmitter power of the Base station's antenna.
\end{abstract}

Keywords: Reuse distance (RD), Base Station (BS), Co-channel Interference (CCI), Transmitter Power (TP), Cell, Frequency Reuse, Adjacent Channel Interference.

\section{Introduction}

\section{Cell:}

A Cell is the basic unit of the cellular system that is commonly known as a hexagon. Each and every cell has a BS that transmits over a cell, because of the constraints inflicted by natural territory and artificial structure. The shape of cell is not exactly hexagonal. The coverage area of cell is called the footprint. The BS simultaneously communicates with multiple mobile devices using single channel (pair of frequencies) per mobile. One frequency is from the Base station to the mobile and other frequency is from the mobile to Base station). Each cell size varies depending on landscape and demand in a specific region. Cells can be added to accommodate growth e.g.; creating new cells by overlaying and splitting the existing cells. This mechanism enhances the capacity of the system. Sectoring the existing cells and thereby using directional antenna can also increase capacity [1]. 


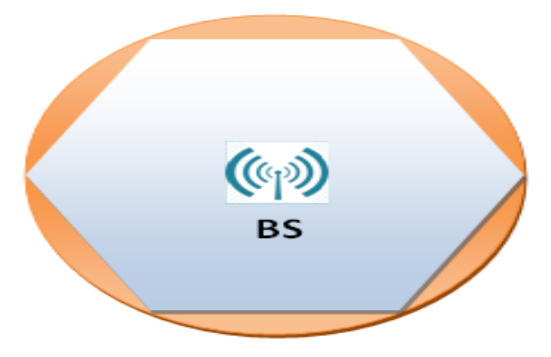

Figure 1. Circular Cell Forming Hexagonal

\section{Cluster:}

A set of cells forms a cluster. Frequencies cannot be reused within a cluster. Frequencies used in one cell cluster can be utilized in another cluster of cells. Many cells in each cluster reduce interference to the system [2].

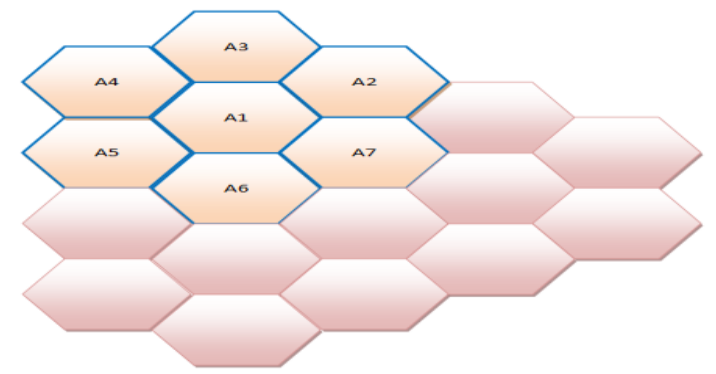

Figure 2. A Seven Cell Cluster

\section{Frequency Reuse}

Frequency reuse is a method of assigning channels to the cellular system [3]. Channels frequencies must be reused because of the unavailability of the spectrum in the cellular band. Cells are assigned group of channels that varied from their neighboring cells. Cells containing same number have the same set of frequencies. The frequency reuse factor is $1 / 7$ if the number of available frequency is 7 that imply that each cell is using 1/7 of available frequencies. Frequency reuse initiates interference into the system. The $2.4 \mathrm{GHz}$ channels contain a vast amount of overlap, which is why some routers only allow you to choose from channels 1, 6 and 11. The use of channel 14 is not permitted in the UK.
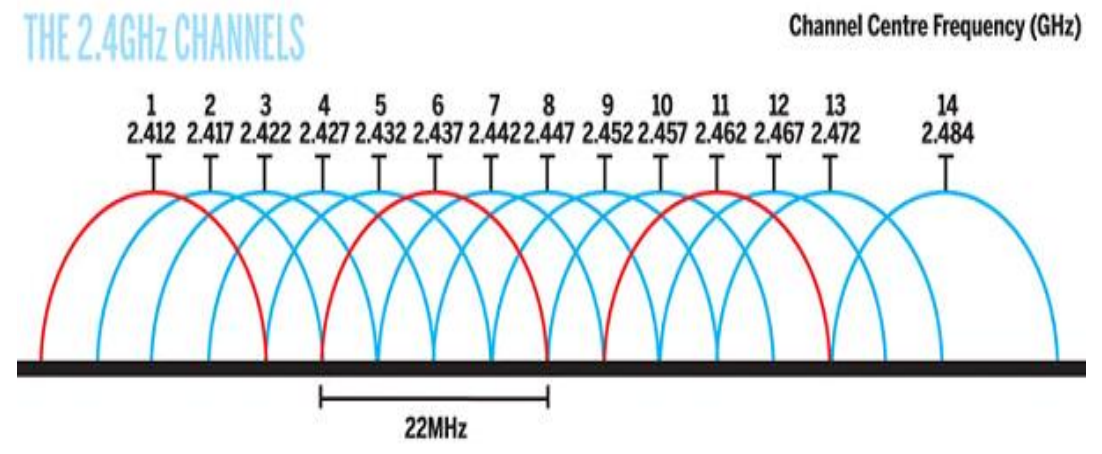

Figure 3. Frequency Distribution of Mobile Communication 


\section{Types of Interference}

In Cellular Communication, we face two types Interference when we want to do Frequency Re-use. They are classified as: Adjacent Channel Interference, Co-Channel Interference.

\section{Adjacent Channel Interference (ACI):}

Adjacent channels are the channels within the RF band that is being used in essence, side-by-side. These adjacent channels overlap each other. When two or more access points using overlapping channels are located near enough to each other than their coverage cells physically overlap and this causes adjacent channel interference. Throughput in a wireless LAN can be severely degraded by adjacent channel interference [4]. It is especially important to pay attention when to locate access points in order to achieve higher throughput in a given area.

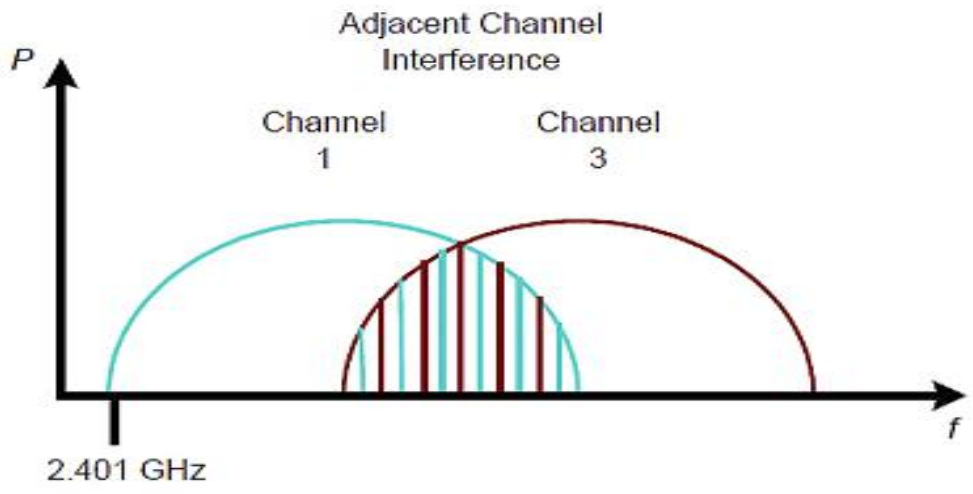

Figure 4. Adjacent Channel Interference

\section{Co-Channel Interference (CCI):}

In cellular system, co-channel means that cell that are reuses distance apart but using the same frequency band. Co-channel interference is cross talk from two different radio transmitters that uses the same frequency width [5].

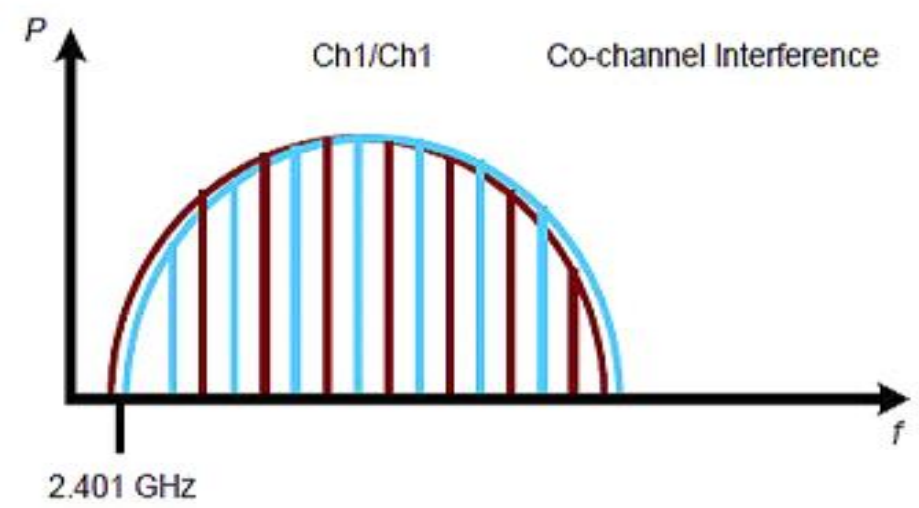

Figure 5. Co-Channel Interference 


\section{Cell Splitting:}

Cell splitting is the process by which a cell is subdivided into smaller cells that contain their own base stations. They have reduced antenna height and transmitting power.

\section{Cell Sectoring:}

A single omnidirectional antenna is replaced by many directional antennas to reduce the co-channel interference in a cellular system and also to provide more channels per unit coverage area.

\section{Cell Sectorization:}

Three or six omnidirectional antennas are placed in place of single omnidirectional antenna at each base station to increase the subscriber capacity of a cellular network.

\section{Reuse distance:}

If two cells are using the same frequency (in different clusters) then the closest distance between the centers of is determined by the selection of the cluster size and the lay-out of the cell cluster is termed as frequency reuse distance.

\section{Related Work}

A number of various approaches have been proposed to reduce frequency reuse distance in mobile communication. In [6] a method dependent on trellis coded modulation (TCM) is offered which enables the system to perform satisfactorily even at reduced levels of C/I. The proposed method gives the pliability to use four or three cell repeat patterns, thus hugely increasing the spectral efficiency of the system. The scheme proposes the use of different TCMs in the distinct co-channel cells. Model results have shown that for a four-cell repeat pattern, the proposed method resulted in a bit error rate of $10-5$ or better, alongside an outage probability of $0.86 \%$.

In [7] the method consists in the use of the average network traffic data and a numbers of measurements taken within a part of a network, along with the signals that are recorded and received from all base stations within reach of a certain given point. The measurement area is divided into polygonal sections, connecting to specific $\mathrm{P}$ measurements and the entire area of such section represents the weight of a given measurement. Eventually, based on the most powerful signal criterion, each $\mathrm{P}$ measurements are assigned to specific network cells. More over it is depended on the differences between signal power value calculated, measurement weights and the presumed distances between channels, values of certain calculated that provide the basis for determining the minimum distances between channels.

In [8] authors offered a wireless multi-hop virtual cellular network (VCN). The first step of transverse to VCN from the existing cellular network $(\mathrm{CN})$ is a foundation of a 2hop VCN. Automatic repeat request (ARQ) is requisite technique in packet transmission. Since the delay time increments in the multi hop communication, the throughput of 2-hop VCN may degrade compared to the conventional 1-hop CN. Even so, the frequency reuse distance of 2-hop VCN may be reduced compared to the conventional 1-hop CN, that the transmit power degrades in the multi hop communication. 


\section{Proposed Work}

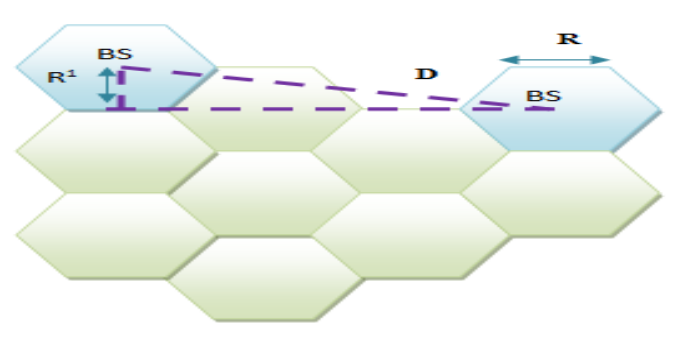

Figure 6. Calculation of Reuse Distance

From the above (figure 6) we can calculate the reuse distance as:

$$
D=\sqrt{\left(\frac{9}{2} R\right)^{2}+\left(\frac{\sqrt{3}}{2} R\right)^{2}}=\sqrt{21} R-4.58 R
$$

Now consider an isotropic point source fed by a transmitter of $P_{t}$ watts. At an arbitrary, large distance $d$ from the source, the radiated power is uniformly distributed over the surface area of a sphere. From the general hypothesis we can say that $\mathrm{R} \simeq \mathrm{d}$. Thus, the received signal power $P_{r}$ at distance $d$ is given by,

$$
P_{r}=\frac{A_{e} G_{t} P_{t}}{4 \pi d^{2}}
$$

Where $A_{e}$ is the effective area overspread by the transmitter, $G_{t}$, is the transmitting antenna gain and $\lambda$ is the wavelength of the electromagnetic wave. The relationship between an effective aperture and the receiving antenna gain $G_{r}$, can be given by,

$$
G_{r}=\frac{4 \pi A_{e}}{\lambda^{2}}
$$

By substituting $A_{e}$ of Equation (2) into Equation (1), we obtain

$$
P_{r}=\frac{G_{r} G_{t} P_{t}}{\left(\frac{4 \pi d}{\lambda}\right)^{2}}
$$

Considering $G_{t}=G_{r}=1$ as free space path loss from equation (3) we can write,

$$
\begin{gathered}
P_{r}=\frac{P_{t}}{\left(\frac{4 \pi d}{\lambda}\right)^{2}} \\
\text { or } P_{t}=P_{r}\left(\frac{4 \pi d}{\lambda}\right)^{2}
\end{gathered}
$$

Taking $P_{r}\left(\frac{4 \pi}{\lambda}\right)^{2}$ as constant $\mathrm{C}$, we can rewrite the equation (4) as $P_{t}=C d^{2}$ (6) 


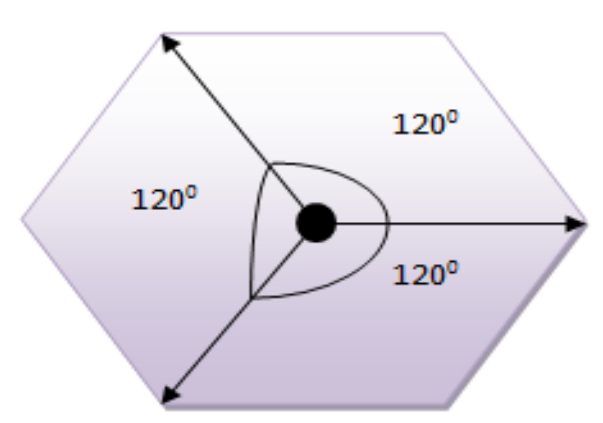

Figure 7. 1200 Sectoring

Now if we use sectoring in a cell, then the total area covered by a cell's antenna will be reduced. Supposed we use sectoring in a cell using $120^{\circ}$ unidirectional antenna as shown in (figure B), so each antenna have to cover only $A_{e} / 3$ of the total cell's area and hence lower power is required in transmitting radio signals, which is $P_{t} / 3$ (It also helps in decreasing interference between co-channels). From equation (6) we can write,

$\frac{P_{t}}{3}=C d^{2}$

or, $P_{t}=C * 3 * d^{2}$

From equation (1) we can write

$P_{t}=C * 3 *\left(\frac{D}{4.58}\right)^{2}$

or, $P_{t}=0.14301 * C * D^{2}$

or, $P_{t}=C_{1} D^{2} \quad$ Taking $\mathrm{C}_{1}=0.14301 * \mathrm{C}$

or $P_{t} \propto D^{2}$

From equation (7) we can conclude that transmitted signal power is directly proportional to the square of reuse distance and earlier we have seen that transmitted signal power varies according to the coverage area of the base station antenna. So when we are sectoring a given cell, antenna's coverage area gets reduced and it also decreases the reuse distance.

\section{Simulation Result}

By using equation 7 , where we have taken $\mathrm{C}=1$ and $\mathrm{C} 1=0.14301$ we have generated this graph, taking $\mathrm{X}$ axis as Reuse Distance and $\mathrm{Y}$ axis as Transmitter Power. From the given below figure it's clear that as we increase the transmitter signal power our reuse distance also gets increased and vice versa. So from this we can conclude that using sectoring we can actually reduce the Reuse distance. 


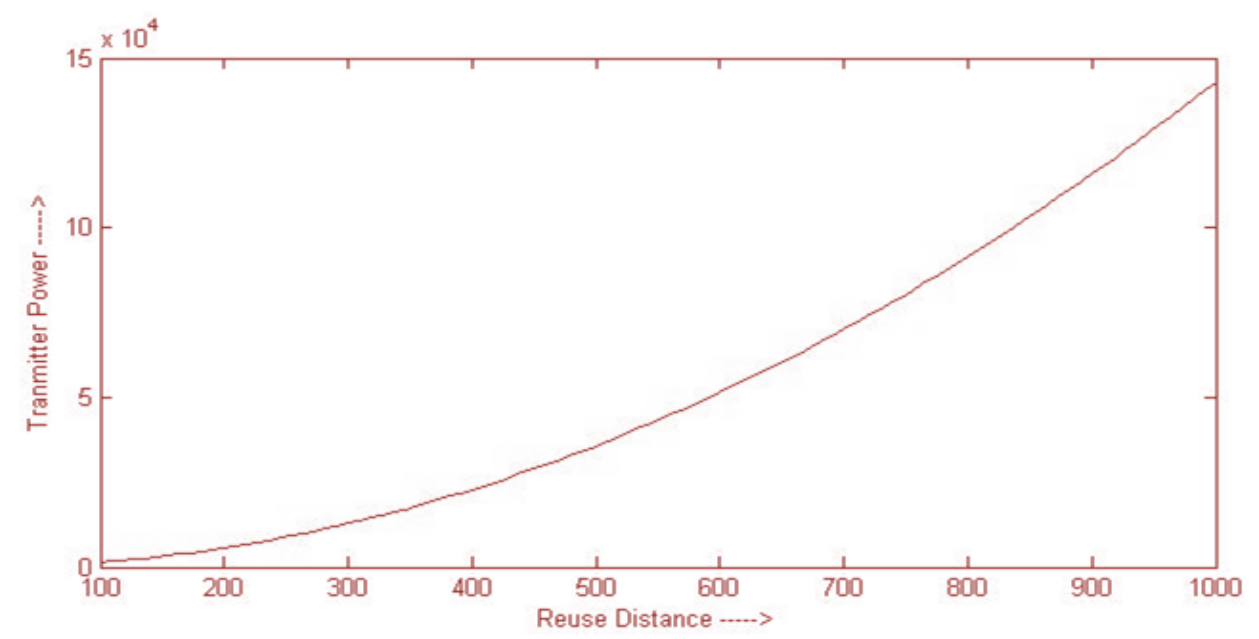

Figure 8. Simulation Result

\section{Conclusion and Future Work}

Our proposed method aims at reducing the reuse distance by reducing the coverage area of a cell which is experienced by sectoring the cell using omnidirectional antenna. This in turn degrades the transmitter antenna's power which in turn decreases the reuse distance brilliantly that is clear from the simulation presented in the above section. In the proposed algorithm a linear trend equation is suitable because it is the most common and trustworthy fit. Future works in this field may include research on channel borrowing from the neighboring cells or sectors. It is worth mentioning here that although the proposed job has been presented considering 1200 sectoring structures yet it would work in any sectoring structure.

\section{References}

[1] Y. Asanuma, “Overlay cell type mobile communication system”, US 08/806,196, (1999).

[2] C. R. Lin, "Adaptive clustering for mobile wireless networks", Dept. of Comput. Sci. \& Inf. Eng., Nat. Chung Cheng Univ., Gerla, M. US8169961 B2, Erteilung, US 12/278,693, PCT/EP2007/051131, (2007).

[3] M. L. Cunningham "Mobile communication system and method employing frequency reuse within a Geroraphical service area", US3898390 A, Erteilung, (1973).

[4] D. Prakash, A. Qing and A. Z. Cengage, "Introduction to Wireless and Mobile system", Second Edition, Ch-13, pp. $303-354$.

[5] L. J. Cimini, "Analysis and Simulation of a Digital Mobile Channel Using Orthogonal Frequency Division Multiplexing", AT\&T Bell Laboratories, vol. 33, no. 7, (1985), pp. 665-675.

[6] R. Bose, "Reducing frequency reuse distance in cellular communications using trellis coded modulation", Dept. of Electr. Eng., Indian Inst. of Technol., Kumar, A. IEEE proceedings communications, (2001); Delhi, India.

[7] "Methods for determination of the minimum distance between frequency channels within pre-selected base station cells", http://www.freepatentsonline.com/EP1389401.html, US6987973 B2, Grant, US 10/478,972, PCT/PL2001/000097, (2006).

[8] E. Kudoh and F. Adachi, "Frequency reuse distance of A 2-hop virtual cellular network", Tohoku Univ., Sendai; print issn: 2163-0771 inspec accession number: 10429123, IEEE, (2008). 


\section{Authors}
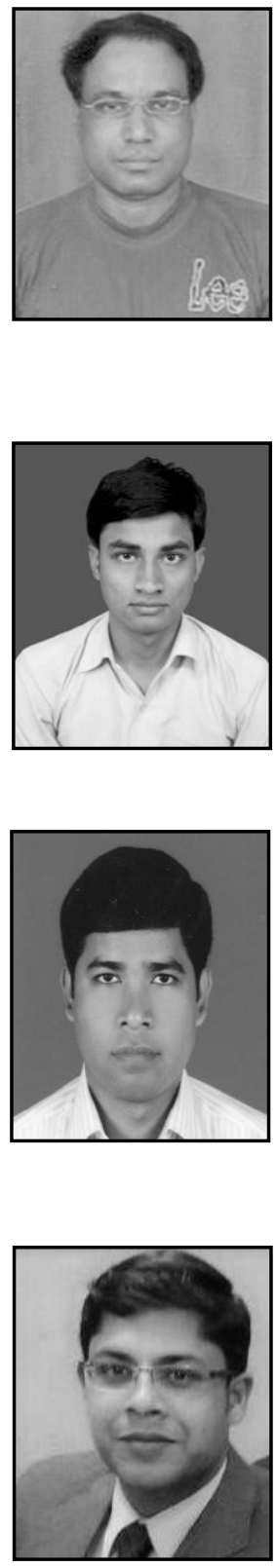

Debabrata Sarddar, Assistant Professor in the Department of Computer Science and Engineering, University of Kalyani, Kalyani, Nadia, West Bengal, INDIA. He has done $\mathrm{PhD}$ at Jadavpur University. He completed his M.Tech in Computer Science \& Engineering from DAVV, Indore in 2006, and his B.E in Computer Science \& Engineering from NIT, Durgapur in 2001. He has published more than 150 research papers in different journals and conferences. His research interest includes wireless and mobile system, Cloud Computing and WSN.

Priyajit Sen is presently pursuing M.Tech in Computer Science and Engineering at the Department of Computer Science and Engineering, University of Kalyani, Kalyani, Nadia, West Bengal, India. He has completed his MCA from Department of Computer Science and Engineering, University of Kalyani, Kalyani, Nadia, West Bengal, India in 2015. His research interest includes Mobile Computing, Wireless Sensor Network and Cloud Computing.

Rajat Pandit is an assistant professor in the Department of Computer Science, West Bengal State University, Barasat, and West Bengal, India. He has completed his M.Tech (IT) from West Bengal University of Technology, West Bengal, India in 2009. He has completed his MCA from University of Jadavpur University, Jadavpur, and West Bengal, India in 2001. His research interest includes Mobile Computing, Wireless Sensor Network and Cloud Computing.

Sougata Chakraborty is a Senior System Engineer at IBM India Private Limited in Kolkata. He completed M. Tech in Computer Science \& Engineering from Jadavpur University in 2011. He had also completed his B.Tech in Information Technology from Murshidabad College of Engineering \& Technology under West Bengal University of Technology in 2008. His research interests include Cloud Computing and Mobile Computing. 